\title{
Health Care and Outcomes
}

\section{Are Family Physicians Good for You? Endogenous Doctor Supply and Individual Health}

\author{
Hugh Gravelle, Stephen Morris, and Matt Sutton
}

Objective. To investigate the impact of family physician (FP) supply on individual health, adjusting for factors that affect both health and FPs' choice of location.

Study Population. A total of 49,541 individuals in 351 English local authorities (LAs). Data Sources. Data on individual health and personal characteristics from three rounds $(1998,1999$, and 2000) of the Health Survey for England were linked to LA data on FP supply.

Study Design. Three methods for analyzing self-reported health were used. FP supply, instrumented by house prices and by age-weighted capitation payments for patients on FP lists, was included in individual-level health regressions along with individual and LA covariates.

Results. When no instruments are used FPs have a positive but statistically insignificant effect on health. When FP supply is instrumented by age-related capitation it has markedly larger and statistically significant effects. A 10 percent increase in FP supply increases the probability of reporting very good health by 6 percent.

Conclusion. After allowing for endogeneity, an increase in FP supply has a significant positive effect on self-reported individual health.

Key Words. Family physicians, health, instrumental variables, doctor supply

Many countries use regulation or financial incentives in an attempt to increase the supply of primary care doctors or family physicians $(\mathrm{FP})$ to areas perceived as underserved. Such policies rest on the premise that increasing the supply of family physicians will improve the health of the individuals in the area. Although the premise is plausible, its evidence base is rather weak. Studies to date, which have been mainly on U.S. state-level data (see, e.g., Shi et al. 2004), suggest that increased supply of FPs improves health. Only two studies examine the relationship between area FP supply and individual health (Shi and Starfield 2000; Laditka 2004). Because of the variations in insurance plans and coverage, modes of service delivery, and contractual 
arrangements in the United States, there is a risk of confounding. Neither the state-nor individual-level studies have allowed for endogeneity of FP supply. If unobserved factors affecting health are correlated with the factors influencing FPs' choice of area, then estimates of the effect of area FP supply on health may be biased.

In this paper, we use a rich multilevel English data set to estimate the effect of area supply of FPs on individual health. The institutional setting in England provides an opportunity to estimate the effect of FP supply on health with less risk of confounding because primary care is almost entirely provided via a tax-financed zero-charge public health service and there was a single national contract for family physicians during the time period of our study. We test for and find FP supply endogeneity, and control for it using instrumental variables.

\section{BACKGROUnd: FPS IN THE NATIONAL HeALTh SERViCE}

The National Health Service (NHS) provides universal tax financed primary and secondary health care. There are no user charges except for drugs provided outside hospital, and because of widely drawn exemptions over 90 percent of these are dispensed without charge. There is a small private sector that provides elective hospital care and is funded by private insurance paid for by employers or individuals.

Individuals register with FPs who provide primary medical services and act as gatekeepers to elective NHS hospital care. FPs in England are selfemployed and work in for-profit partnerships with a modal size of four FPs. During the time-period covered by our study, FP income was determined by a national contract whose main element was a capitation fee for each patient on the FP's list.

From the founding of the NHS in 1948 FPs' location decisions were regulated by a national body-the Medical Practices Committee. Its aim was to reduce inequalities in the regional distribution of FPs. It attempted to do so by restricting entry into areas it designated as already adequately supplied with

Address correspondence to Hugh Gravelle, Ph.D., National Primary Care Research and Development Centre, Centre for Health Economics, University of York, YO10 5DD, UK. Email: hg8@york.ac.uk. Stephen Morris, Ph.D., is with the Health Economics Research Group, Brunel University, Middlesex, UK and also with the National Primary Care Research and Development Centre, University of Manchester, Manchester, UK. Matt Sutton, Ph.D., is with the Health Economics Research Unit, University of Aberdeen, U.K. 
FPs. These entry restrictions, and incentives to encourage FPs to practice in under-doctored areas, were unsuccessful in achieving an equal distribution (Hann and Gravelle 2004).

\section{METHODS}

There are a number of ways in which increased FP supply might improve health. We argue that greater FP supply in an area reduces access costs for patients thereby increasing utilization (Escarce 1992), and greater utilization, at any given level of health need, improves health. Moreover, there are no monetary barriers to primary care in the NHS and all patients have a right to be placed on a family practice list. Thus, more FPs per head in an area will also result in practices with more FPs per patient on their list. There is some evidence that family practices with fewer patients per FPs have longer consultations (Campbell, Ramsay, and Green 2001) and that longer consultations produce better outcomes (Wilson and Childs 2002).

We do not attempt to identify the pathways by which FP supply affects health. We estimate a "reduced form" effect of FP supply on individual health by estimating a two-stage model. We first use an area-(local authority [LA]) level regression of FP supply on area-level variables to predict FP supply. We then estimate an individual-level regression of self-reported health on a rich set of individual covariates, predicted FP supply, and area variables.

\section{Individual Data}

Individual-level data on health and socioeconomic status were obtained from the Health Survey for England (HSE) (Sproston and Primatesta 2003). The HSE is a nationally representative survey of individuals aged 2 years and older living in England. A new sample is drawn each year and information is collected on health, demographic, and socioeconomic factors via interviews. We use three rounds of the HSE from 1998 to 2000, yielding a total of 49,541 observations.

Individual-level socioeconomic covariates in the health equation include age, gender, equivalized household income, social class of head of household (seven categories), highest educational qualification attained (seven categories), ethnic group (nine categories), and marital status (five categories).

We include two year indicators to allow for any possible trend in reported health and 11 month interview indicators to remove the effects of 
any seasonal factors. We also use an indicator of whether information for children was obtained from a proxy respondent.

We imputed missing items for covariates. For continuous variables missing values were imputed using the linear prediction from a regression of the variable on the other covariates. For categorical variables, missing values are assigned to the omitted category. To allow for the possibility that items are not missing at random, we include dummy variables to indicate item nonresponse.

\section{Health Measures}

The dependent variable in the health equation is self-reported general health status. Respondents are asked: "How is your health in general? Would you say it was: 'Very good,' 'Good,' 'Fair,' 'Bad,' 'Very bad'?' Self-assessed health measures have been shown to be good predictors of subsequent mortality (Idler and Benyammi 1997) for all socioeconomic groups (Burström and Fredlund 2001) at the individual level, to be strongly associated with mortality rates at the area level (Kyffin, Goldacre, and Gill 2004), and to reflect more objective health indicators of a range of specific health conditions (Sutton et al. 2002). We construct three health measures from the answers to this question and estimate three health models, thereby providing a functional form robustness check.

Self-Assessed General Health. We estimate an ordered probit model for the responses $h_{i a t}^{1}=1,2,3,4$, and 5 to the self-assessed general health question

$$
\begin{aligned}
& h_{i a t}^{1}=j \text { if } \mu_{j-1}<; h_{i a t}^{1 *}<; \mu_{j}, \text { for } j=1,2, \ldots, 5, \\
& \mu_{0}=-\infty, \mu_{5}=+\infty
\end{aligned}
$$

where $h_{i a t}^{1 *}$ is latent health for individual $i$ in area $a$ in year $t . h_{i a t}^{1 *}$ is determined by

$$
\begin{aligned}
h_{i a t}^{*} & =\beta_{0}+\beta_{1} x_{i a t}+\beta_{2} x_{i a}+\sum_{s} \beta_{3 s} D_{s}+\sum_{k} \beta_{4 s} M_{k}+\gamma g_{a t-1}+\varepsilon_{i a t}^{1}, \\
\varepsilon_{i a t}^{1} & \sim N(0,1)
\end{aligned}
$$

where $x_{i a t}, x_{a t}$ are individual- and area-level variables, $g_{a t-1}$ is the logarithm of area FP supply, and $D_{s}, M_{k}$ are year and month of HSE interview dummies. 
Discrete Attributed EQ-5D Score. The 1996 HSE contains EQ-5D health instrument (EuroQol Group 1990) ${ }^{1}$ scores for 16,047 respondents as well as their self-reported general health status. We computed the mean EQ-5D score for the individuals in the $j$ th self-assessed general health categories in the $1996 \mathrm{HSE}$ and assigned it as the health score for individuals in category $j$ in the 1998-2000 rounds of the HSE. We treat this measure $\left(h^{2}\right)$ as a cardinal variable and estimate the health model by least squares regression specified as in (2), with $h_{\text {iat }}^{2}$ replacing $h_{\text {iat }}^{*}$.

Continuous Attributed EQ-5D Score. We also attributed a continuous cardinal EQ-5D score $h_{\text {iat }}^{3}$ for each individual. ${ }^{2}$ We used the 1996 HSE data on EQ-5D scores and self-assessed general health to set the cut points for the self-assessed health categories (the $\mu$ s in [1]) (see Supplementary Material Appendix SA). We then estimate the health model by interval regression specified as in (2) with $h_{\text {iat }}^{3}$ replacing $h_{\text {iat }}^{*}$.

\section{Area Data}

We constructed area-level FP supply variables for 1997, 1998, and 1999 using the General Medical Services (GMS) Statistics database. ${ }^{3}$ We computed FP supply at the LA level. England is divided into $354 \mathrm{LAs}$, which have a mean resident population of 141,235 (range 2,149-1,010,427). In the regressions, we use the logarithm of the number of whole-time equivalent FPs per 1,000 residents as the FP supply measure. Because the FP supply variable is measured at September of each year and the HSE interviews are collected throughout the year, we use the previous year's FP supply in the health models. Experiments with longer lags of FP supply yielded very similar results, reflecting the high temporal correlation of area-level FP supply.

The individual-level models also contain 54 LA variables measuring area socioeconomic status and accessibility to other health services. Measures of the accessibility of other health service were taken from the Allocation of Resources to English Areas (AREA) data set (Sutton et al. 2002). Variables included distances to hospitals, waiting times, beds, and numbers of specialists. Additional area-level data were obtained from the Neighborhood Statistics data set maintained by the Office for National Statistics. ${ }^{4}$ They included crime rates and measures of socioeconomic deprivation. Except for FP supply, the LA variables are time invariant. We also used eight dummy variables for the Government Office Regions within which LAs are situated. 


\section{Instrumental Variables for FP Supply}

The use of a large number of individual- and area-level covariates in the health regressions reduces, but may not eliminate, the risk of endogeneity bias affecting the estimated effect of area FP supply on health. The bias could be positive or negative. If FP supply depends in part on the amenities and attractiveness of an area, and these factors are not captured by the area-level covariates in the health regression and are also correlated with individual health, then the estimated effect of FP supply is biased upwards. But FP supply is also plausibly affected by income. The FP contract and local health authority policies produce financial and other incentives for FPs locating in areas with more deprived patients. If more deprived patients are less healthy, conditional on the individual and area covariates in the health regression, and FP supply is greater, conditional on these covariates, in areas with more deprived patients, then the estimated effect of FP supply on health is biased downward.

We instrument FP supply using two LA-level variables. The first is an index of local area house prices in $2000 .{ }^{5}$ Given the large number of individual and other area variables in the health regression, it is unlikely that area house prices correlate with individual health. If local house prices are mainly driven by differences in supply due to planning restrictions, then we expect higher prices to reduce the supply of FPs in an area because there is no local cost-ofliving adjustment in the national FP contract. House prices may also be affected by area amenity or attractiveness. But we include a large number of area covariates in the FP supply and health models and so expect that, conditional on these covariates, higher house prices will reduce FP supply. House price indices are available for apartments, terraced houses, detached houses, and semidetached houses. Experiments showed that the semidetached house price index had the most significant coefficient in the FP supply model conditional on the other variables and we use this as the instrument.

The second instrument is age-related capitation payment per head of population. For each patient on their list FPs receive a capitation fee which increases with the age band $(0-64,65-74$, and $75+)$ of the patient. Hence, we expect to find more FPs in areas where the population generate higher age related capitation payments, all else equal. The Medical Practices Committee was also less likely to restrict entry into areas with an older population (Medical Practices Committee 1998). Although age is negatively correlated with health at the individual level, we include a gender varying cubic function of the individual's age in the health regressions, so that it is plausible that any unobservable factors affecting individual health are uncorrelated with the age of 
the LA population. As an alternative flexible age specification, we also reran the regressions using 20 -year age band indicators.

We computed the weighted average age related capitation payment per person in area $a$ as $\sum_{k=1}^{3} \frac{N_{k a}}{N_{a}} Q_{k}$, where $N_{k a}$ is the number of people in LA $a$ in age band $k, N_{a}$ the total population of LA $a$, and $Q_{k}$ is the capitation payment for age band $k$ (Department of Health 2000). The proportion of the LA population in each age band was obtained for 2000 from the AREA dataset (Sutton et al. 2002).

\section{Estimation}

At stage 1, we run separate annual OLS regressions for ln FP supply at LA level

$$
g_{a t}=\alpha_{0 t}+\alpha_{1 t} x_{a}+\alpha_{2 t} v_{a}+u_{a t}
$$

where $g_{a t}$ is $\ln$ FPs per 1,000 population in area $a$ at year $t, x_{a}$ are LA covariates and $v_{a}$ are the potential instruments (semidetached house price and age-related capitation). Because there is no house price data for three LAs (City of London, Isle of Wight, and the Isles of Scilly), the FP regressions uses 351 LAs. We use the same time invariant instruments in each of the separate FP regressions. The instruments are valid if they are good predictors of each year's FP supply and uncorrelated with the error in the health regression. The latter requires that not only is predicted FP supply in year $t-1$ uncorrelated with the year $t$ errors in the health equation but that it should be uncorrelated with the errors for all 3 years. ${ }^{6}$

We estimate the stage 2 health model at the individual level using predicted LA ln FP supply as the measure of instrumented FP supply, plus individual covariates, plus all the LA variables in the ln FP supply model except the instruments. We drop 112 individuals who are in the three LAs with no house price data. A total of 49,541 individuals are left in the individual-level sample.

We report results from health models using three sets of instruments: age-related capitation only (including house prices in the health regression), house prices only (including age-related capitation in the health regression), and both instruments.

In the FP supply models we weight each LA observation by the size of the resident population, and calculate robust standard errors. In the uninstrumented health equation we report standard errors that are adjusted for clustering within LAs. 
In the instrumented health equation the standard errors on predicted $\ln$ FP supply must be corrected to account for the fact that ln FP supply is an estimate. Karaca-Mandic and Train (2003) note that the Murphy and Topel (1985) estimator is inappropriate when there are differences in the sample sizes in the first and second stage models. We therefore use a bootstrap procedure:

(i) Draw a sample of 351 LAs with replacement from the area-level data set.

(ii) Estimate the ln FP supply equations for each of the 3 years-1997, 1998, and 1999.

(iii) Estimate the individual-level health equation using predicted ln FP supply plus the individual- and area-level covariates. The individual observations are weighted by the number of times the LA in which an individual lives was drawn in the first stage bootstrap sample.

The reported standard error of the instrumented ln FP supply coefficient is the standard deviation of the estimated coefficients on In FP supply from 200 replications of this procedure.

We check for the endogeneity of FP supply in the health model using an augmented regression (StataCorp 2005, p. 504.). We repeat the estimation procedure described above but as well as including predicted ln FP supply we also include actual ln FP supply. If the coefficient on predicted ln FP supply is significantly different from zero then, under the maintained assumption that the instruments are valid, FP supply is endogenous and the IV estimates are preferred.

In addition to checking that the house price and capitation instruments are significant in the first stage FP regression, we perform two other tests of the instruments. First, because we have two instruments we can undertake an over identification test with a bootstrap version of the Hausman test of the difference between the estimated coefficient on $\ln$ FP supply using both instruments and using each instrument on its own.

Second, we construct a version of the Dranove and Meher (1994) "antitest" for instrumental variables. Dranove and Meher (1994) criticize instruments, such as temperature, hotel receipts, and local colleges, used in studies of the effect of physician supply on the volume of medical care. They show that when these variables are used to instrument physician supply they produce the obviously absurd result that increased supply leads to more childbirths. We carry out an analogous "anti-test" for the validity of our instruments. We used instrumented and noninstrumented $\ln \mathrm{FP}$ area supply to explain an individual- 
level variable that cannot be determined by FP supply: ethnicity. Using the same two-stage and one-stage methods as for the health models, we estimated a probit model for individual ethnicity ( $=1$ if white, $=0$ if nonwhite). The explanatory variables and the IVs were the same as those in health model (except that we drop ethnicity as an explanatory variable and do not replace it with health).

\section{RESULTS}

The mean number of FPs per 1,000 patients over the 3 years of 1997-1999 was 0.502 (standard deviation [SD] 0.04). FP supply in LAs was highly correlated over time (correlation 0.955 for 1997 and 1998, 0.917 for 1998 and 1999, and 0.892 for 1997 and 1999). The top part of Table 3 contains summary statistics for the health variables. As is usual with self-reported health surveys a high proportion (over 75 percent) of the sample report that they are in good or very good health. A full set of summary statistics is in Supplementary Material Appendix SB.

The coefficients on the instruments in first-stage least squares FP supply equations for each year are in Table 1. The full results for both the FP supply and health models are in Supplementary Material Appendix SB. As expected, ln FP supply is negatively correlated with house prices and positively correlated with the age-related capitation payments. A 1 SD increase in capitation

Table 1: Impact of the Instruments on FP Supply Measure (ln FPs per 1,000 Population)

\begin{tabular}{|c|c|c|c|c|c|c|}
\hline & \multicolumn{2}{|c|}{1997} & \multicolumn{2}{|c|}{1998} & \multicolumn{2}{|c|}{1999} \\
\hline & $\beta$ & $t$ & $\beta$ & $t$ & $\beta$ & $t$ \\
\hline Average capitation payment & 0.071 & 3.8 & 0.071 & 3.7 & 0.068 & 3.8 \\
\hline House price index & -0.023 & -3.5 & -0.023 & -3.3 & -0.029 & -4.2 \\
\hline$N$ & \multicolumn{2}{|c|}{351} & \multicolumn{2}{|c|}{351} & \multicolumn{2}{|c|}{351} \\
\hline Adjusted $R^{2}$ & \multicolumn{2}{|c|}{0.6298} & \multicolumn{2}{|c|}{0.6010} & \multicolumn{2}{|c|}{0.5143} \\
\hline$F$-test average capitation payment $=0[p$-value $]$ & \multicolumn{2}{|c|}{$14.67[.0002]$} & \multicolumn{2}{|c|}{$13.85[.0002]$} & \multicolumn{2}{|c|}{$14.21[.0002]$} \\
\hline$F$-test house price index $=0[p$-value $]$ & \multicolumn{2}{|c|}{$12.16[.0006]$} & \multicolumn{2}{|c|}{$11.17[.0009]$} & \multicolumn{2}{|c|}{$17.40[<.0001]$} \\
\hline$F$-test both instruments $=0[p$-value $]$ & \multicolumn{2}{|c|}{$12.98[<.0001]$} & \multicolumn{2}{|c|}{$12.10[<.0001]$} & \multicolumn{2}{|c|}{$15.29[<.0001]$} \\
\hline
\end{tabular}

Population weights used in all models. Fifty-four additional area-level covariates are included in each model including deprivation measures, crime rates, hospital supply measures, and eight regional dummy variables.

FP, family physician. 
payment has an effect on $\ln$ FP supply that is three times greater than that of a $1 \mathrm{SD}$ increase in house prices. Local house prices may have less effect on FP supply because FPs need not live in the LAs in which their practices are situated.

Because FP supply at the area level is highly correlated across the 3 years and the instruments used for each of the separate FP regressions do not vary over time, the coefficients on the instruments are very similar across the 3 years. In all years the instruments are individually and jointly significant predictors of ln FP supply conditional on the comprehensive set of covariates. The $F$-values on the instruments comfortably exceed 10, which is often taken as a critical value below which one should be concerned about weak instruments (Staiger and Stock 1997).

Table 2 reports the coefficients on instrumented ln FP supply from the health regressions, which also contained individual demographic and socioeconomic covariates and the 54 area variables from the ln FP supply regressions. Twelve sets of coefficients are reported, for the three health measures, and for actual ln FP supply and predicted ln FP supply using the two instruments separately and jointly. Regressions using dummies for 20 -year age categories, instead of cubic functions of age, produced very similar estimates of the effect of FPs on health (Supplementary Material Appendix Table SB6).

The effects of $\ln$ FP supply are consistent across the three different health measures. In all cases ln FP supply has a positive impact on health status. Compared with the non-IV models, the IV models have a much greater impact of ln FP supply on health. In the models where the house price index is the only instrument, ln FP supply is not significant at the 5 percent level. The effect of ln FP supply is greatest, and has the highest statistical significance, when age-related capitation payments are used as the instrument. Using agerelated capitation as the only instrument increases the estimated effect of $\ln$ FP supply 10 -fold compared with the uninstrumented estimate. Note also that the proportionate differences in coefficients across health variables for a given measure of ln FP supply are similar across non-IV and IV models. For each health measure the relative magnitudes of the effects of each measure of $\ln \mathrm{FP}$ supply (uninstrumented and instrumented) are similar.

Neither the magnitude nor the significance of the coefficient on predicted $\ln$ FP supply are affected by including actual ln FP supply in the health regression, suggesting that FP supply is indeed endogenous (Supplementary Material Appendix Table SB7).

The instruments pass the over identification tests: the coefficients on ln FP supply from the model with both instruments are not significantly different 


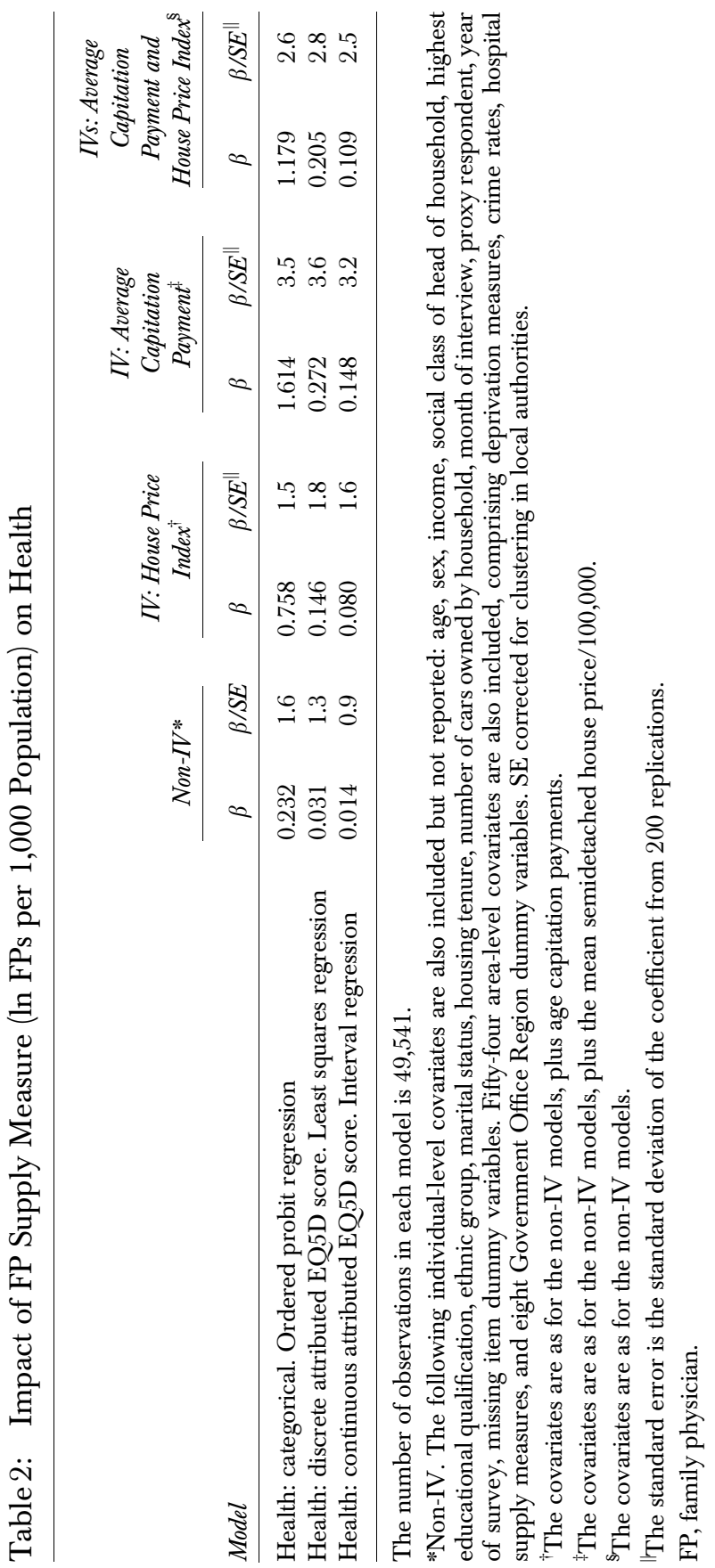


Table 3: Effect of Changes in FPs, Age, and Income on Probabilities of Self-Reported Health Categories

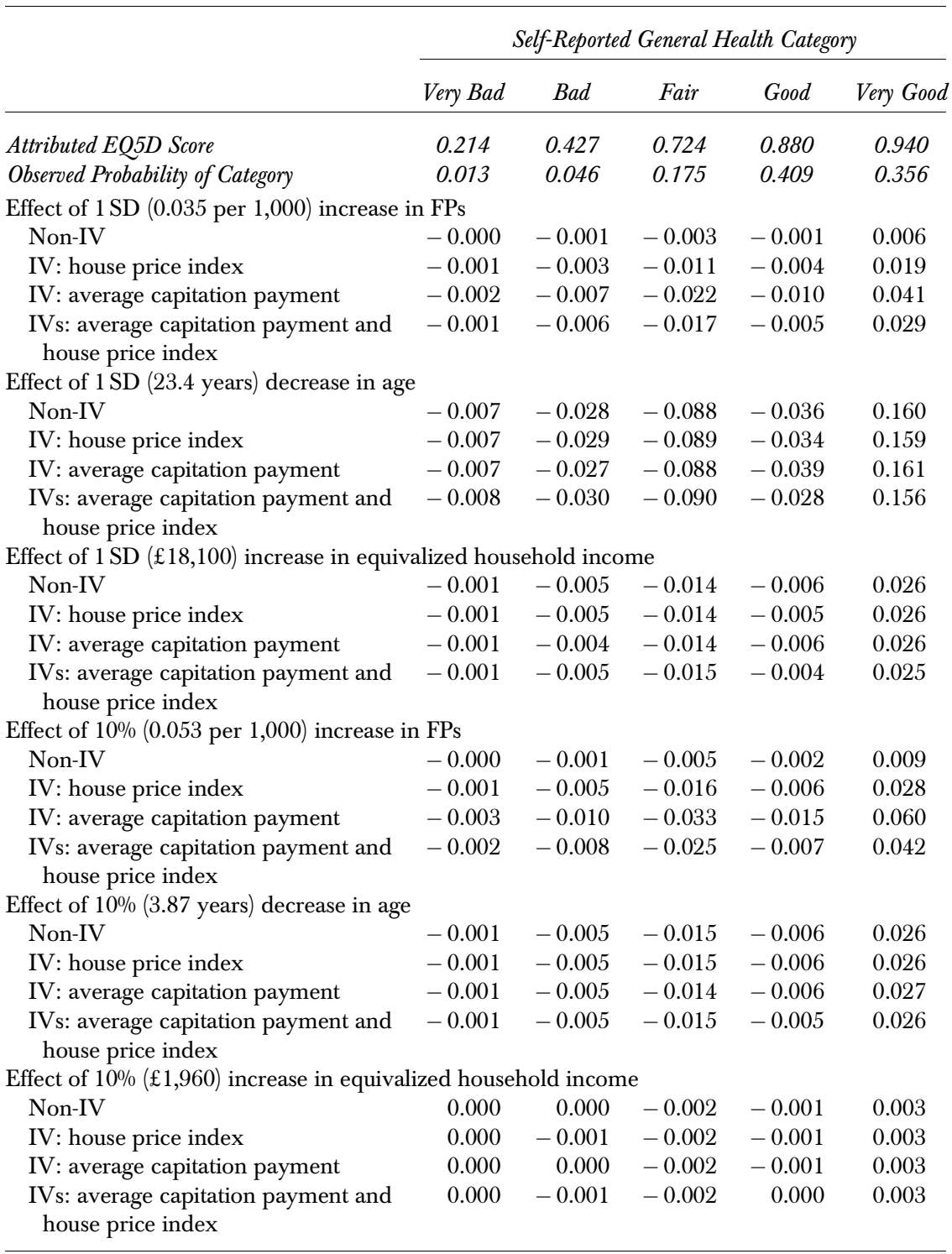

From coefficients of ordered probit health models. Evaluated at means of FP supply and covariates. $\mathrm{FP}$, family physician. 
Table 4: Percentage Change in Health Due to Changes in FP Supply per 1,000, Age, and Income

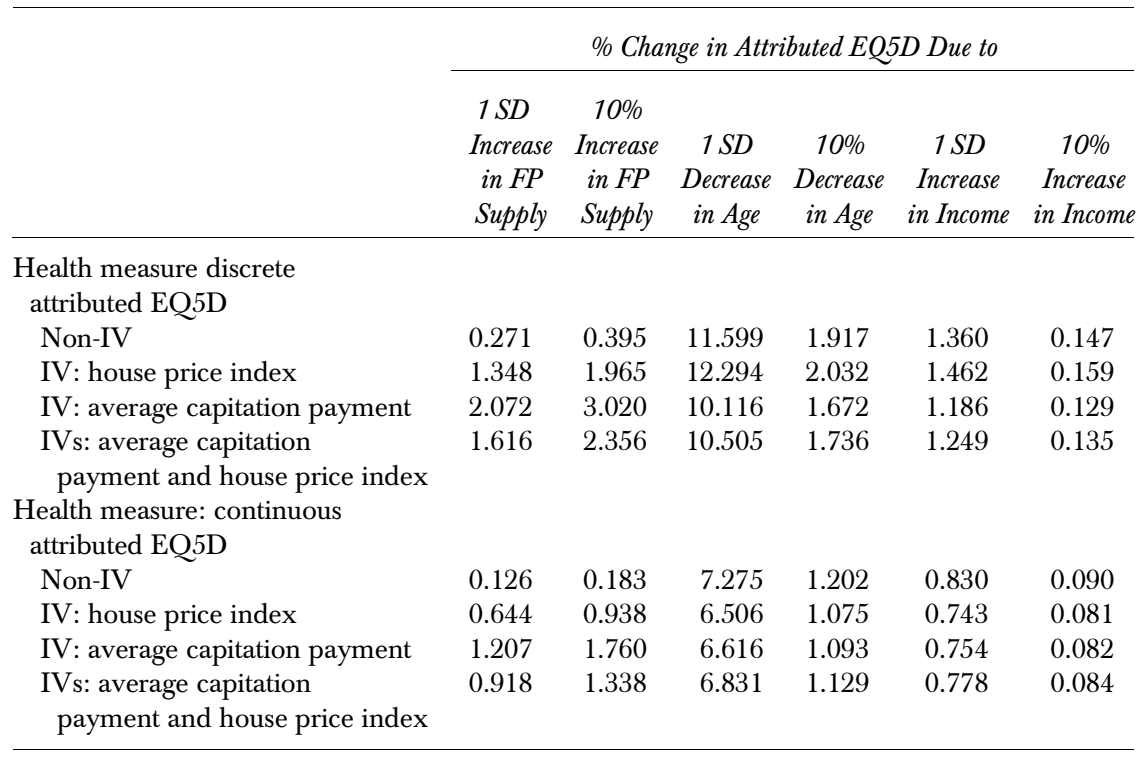

From coefficients of OLS and interval regression models. Evaluated at means of FP supply and covariates.

FP, family physician.

from those models using only one of the instruments (Supplementary Material Appendix Table SB8). The instruments also pass the Dranove and Meher anti-test: ln FP supply is insignificant in explaining individual ethnicity whether FP supply is instrumented with both IVs, or with each separately, or is not instrumented (Supplementary Material Appendix Table SB9).

We illustrate the magnitude of the effects of FP supply on the probability of each health category using the estimated coefficients from the ordered probit $h^{1}$ model with FP supply and all covariates evaluated at their mean values. Table 3 compares the effects of 10 percent and $1 \mathrm{SD}$ changes in FP supply per 1,000 , age, and income. There has been relatively little redistribution of FPs across areas but the average annual growth rate in FP supply between 1995 and 2004 was 1.5 percent. Income and age are much more variable across individuals and income grew at an average annual rate of around 2 percent over this period.

A 1 SD increase in income increases the probability of very good health by .026 for all versions of the health model, whereas the estimated effect of a 
$1 \mathrm{SD}$ increase in FP is to increases the probability of very good health by .041 with the capitation instrument. A reduction in age of 10 percent (3.87 years) increases the probability of very good health by around .026, which is less than half the effect of a 10 percent increase in FP supply estimated using the agerelated capitation instrument.

Table 4 reports the percentage change in the discrete and continuous attributed EQ-5D health measures from changes in FP supply, age, and equivalized income. With the capitation instrument, the effect of a $1 \mathrm{SD}$ increase in FP supply is 1.6-1.75 times as great as that of a $1 \mathrm{SD}$ increase in income, and a 10 percent increase in FP supply has an effect 1.6-1.8 times as great as a 10 percent decrease in age.

\section{CONCLUSION}

Using a multilevel data set with rich information on individuals and areas, we find that area FP supply is positively associated with individual self-assessed health. If no allowance is made for the endogeneity of FP supply, the effect is not statistically significant. We test for and find endogeneity bias. Using instruments (house prices and age-related capitation per head of population) for FP supply yields markedly larger estimates, which are statistically significant when the instrument is age related capitation. The FP contract contains payments for FPs locating in areas with more deprived patients. If more deprived patients are less healthy, conditional on the individual and area covariates in the health regression, and FP supply is greater, conditional on these covariates, in areas with more deprived patients, then the estimated effect of FP supply on health is biased downwards, as is suggested by comparison of the IV and nonIV models.

When FP supply is instrumented by age-related capitation, a $1 \mathrm{SD}$ increase in FP supply increases the probability of reporting very good health by 4.1 percent. The results are robust to transformations of the health variable and to alternative specifications of the effects of individual age on health.

Our study has two weaknesses. First, our instrumental variables are invalid if they are correlated with individual health. We use a rich set of individual- and area-level covariates, which reduces this risk but cannot eliminate it: any use of instrumental variables rests on the uncheckable assumption that, conditional on the covariates, the instruments are uncorrelated with unobservable factors affecting the dependent variable. 
Second, we estimate a reduced form effect of FP supply on health and so do not provide any evidence on how the effect is achieved: by reduced access costs increasing the number of consultations, or by FPs having shorter lists and so more time to provide longer consultations or to invest in other activities improving the quality of care. Nonetheless, the results provide support for the view that improved primary care provision in the form of increased FP supply can improve individual health.

\section{ACKNOWLEDGMENTS}

Funding from the Department of Health to NPCRDC and from the Chief Scientist Office to HERU is acknowledged. The views expressed are those of the authors and not necessarily those of the funders. We are grateful to Jenny Roberts, Martin Roland, and Frank Windmeijer for helpful suggestions and for comments received at the York Seminars in Health Econometrics, and the Social Dimensions of Health Institute at Dundee.

\section{NOTES}

1. The EQ-5D is a descriptive system that provides a profile of a person's health status to which a cardinal index for U.K. population has been attached (Dolan 1997). The index has an upper limit of 1 , corresponding to full health. Death is scored as 0 and states regarded as worse than death have negative scores.

2. See Supplementary Material Appendix SA.

3. See http://www.primary-care-db.org.uk/

4. See http://www.neighbourhood.statistics.gov.uk/home.asp

5. See http://www.landreg.gov.uk/propertyprice/interactive/ppr_ualbs.asp

6. The instrument validity tests and exogeneity tests we run do not depend on the method for generating the instruments.

\section{REFERENCES}

Burström, B., and P. Fredlund. 2001. "Self Rated Health: Is It as Good a Predictor of Subsequent Mortality among Adults in Lower as Well as in Higher Social Classes?" Journal of Epidemiology and Community Health 55: 836-40.

Campbell, J. L., J. Ramsay, and J. Green. 2001. "Practice Size: Impact on Consultation

Length, Workload, and Patient Assessment of Care." British Journal of General Practice 51: 644-50. 
Department of Health. 2000. Statement of Fees and Allowances Payable to General Medical Practitioners in England and Wales. London: Department of Health.

Dolan, P. 1997. "Modelling Valuations for EuroQol Health States." Medical Care 35: 1095-108.

Dranove, D., and P. Meher. 1994. "Physician Induced Demand for Childbirths." Journal of Health Economics 13: 61-73.

Escarce, J. 1992. "Explaining the Association between Surgeon Supply and Utilisation." Inquiry 29: 403-15.

EuroQol Group. 1990. "EuroQol-A New Facility for the Measurement of HealthRelated Quality of Life." Health Policy 16: 199-208.

Hann, M., and H. Gravelle. 2004. "The Mal-Distribution of General Practitioners in England and Wales 1974-2003." British Journal of General Practice 54: 894-8.

Idler, E. L., and Y. Benyammi. 1997. "Self-Rated Health and Mortality: A Review of Twenty-Seven Community Studies.” Journal of Health and Social Behaviour 38: 2137.

Karaca-Mandic, P., and K. Train. 2003. "Standard Error Correction in Two-Stage Estimation with Nested Samples." Econometrics Journal 6: 401-7.

Kyffin, R. G. E., M.J. Goldacre, and M. Gill. 2004. "Mortality Rates and Self Reported Health: Database Analysis by English Local Authority Area." British Medical Journal 329: 887-8.

Laditka, J. N. 2004. "Physician Supply, Physician Diversity, and Outcomes of Primary Health Care for Older Persons in the United States." Health and Place 10: 231-44.

Medical Practices Committee. 1998 "Notes of Guidance for Health Authorities" [accessed December 1998]. Available at http://www.dh.gov.uk/PublicationsAndStatistics/Publications/PublicationsPolicyAndGuidance/PublicationsPolicyAndGuidanceArticle/fs/en?CONTENT_ID=4083566\&chk=m4Yg7s

Murphy, K., and R. Topel. 1985. "Estimation and Inference in Two-Step Econometric Models." Journal of Business and Economic Statistics 3: 370-9.

Shi, L., J. Macinko, B. Starfield, J. Xu, J. Regan, R. Politzer, and J. Wulu. 2004. "Primary Care, Infant Mortality, and Low Birth Weight in the States of USA." Journal of Epidemiology and Community Health 58: 374-80.

Shi, L., and B. Starfield. 2000. "Primary Care, Income Inequality, and Self-Rated Health in the Untied States: A Mixed-Level Analysis." International Journal of Health Services Research 30: 541-55.

Sproston, K., and P. Primatesta, (eds.) 2003. Health Survey for England 2002. London: The Stationery Office.

Staiger, D., and J. Stock. 1997. "Instrumental Variables Regression with Weak Instruments." Econometrica 65: 557-86.

StataCorp. 2005. Stata Statistical Software: Release 9.0. Reference Manual A-J. College Station, TX: Stata Corporation.

Sutton, M., H. Gravelle, S. Morris, A. Leyland, F. Windmeijer, C. Dibben, and M. Muirhead. 2002 "Allocation of Resources to English Areas: Individual and Small Area Determinants of Morbidity and Use of Health Care.” Report for Department of Health Information and Statistics Division, Common Services Agency, Scotland. 
Wilson, A., and S. Childs. 2002. "The Relationship between Consultation Length, Process and Outcomes in General Practice: A Systematic Review." British Journal of General Practice 52: 1012.

\section{SUPPLEMENTARY MATERIAL}

The following supplementary material for this article is available online:

Appendix SA. Attribution of Continuous EQ-5D Score.

Appendix SB. Additional Tables.

Table SB1. Summary Statistics.

Table SB2. Full Results for FP Supply Equations.

Table SB3. Impact of FP Supply on Self-Reported General Health. Regression Model: Ordered Probit.

Table SB4. Impact of FP Supply on Cardinalized Self-Reported General Health. Regression Model: Interval Regression.

Table SB5. Impact of FP Supply on Mean EQ5D Score by Self-Reported General Health Category. Regression Model: OLS.

Table SB6. Impact of FP Supply Measure (ln FPs per 1,000 population) on Health, with Age Categories.

Table SB7. Augmented Regression Test for Endogeneity: Impact of Predicted ln FP Supply Measure on Health.

Table SB8. Over Identification Tests: Difference in Coefficients on Predicted ln FP Supply Measures Using Both IVs and Each IV Individually.

Table SB9. Dranove and Meher (1994) "Anti-Test" of IV Validity: Impact of FP Supply Measure (ln FPs per 1,000 Population) on Ethnicity.

This material is available as part of the online article from: http:// www.blackwell-synergy.com/doi/abs/10.1111/j.1475-6773.2007.00823.x (this link will take you to the article abstract).

Please note: Blackwell Publishing is not responsible for the content or functionality of any supplementary materials supplied by the authors. Any queries (other than missing material) should be directed to the corresponding author for the article. 